Pacific

Journal of

Mathematics

SYMMETRIES OF REAL CYCLIC $p$-GONAL RIEMANN SURFACES

Antonio F. Costa And Milagros Izquierdo

Volume $213 \quad$ No. 2

February 2004 


\title{
SYMMETRIES OF REAL CYCLIC $p$-GONAL RIEMANN SURFACES
}

\author{
Antonio F. Costa and Milagros Izquierdo
}

\begin{abstract}
A closed Riemann surface $X$ which can be realised as a $p$-sheeted covering of the Riemann sphere is called $p$-gonal, and such a covering is called a $p$-gonal morphism. A $p$-gonal Riemann surface is called real $p$-gonal if there is an anticonformal involution (symmetry) $\sigma$ of $X$ commuting with the $p$-gonal morphism. If the $p$-gonal morphism is a cyclic regular covering the Riemann surface is called real cyclic $p$-gonal, otherwise it is called real generic $p$-gonal. The species of the symmetry $\sigma$ is the number of connected components of the fixed point set Fix $(\sigma)$ and the orientability of the Klein surface $X /\langle\sigma\rangle$. In this paper we find the species for the possible symmetries of real cyclic $p$-gonal Riemann surfaces by means of Fuchsian and NEC groups.
\end{abstract}

\section{Introduction.}

A closed Riemann surface $X$ which can be realised as a $p$-sheeted covering of the Riemann sphere is called $p$-gonal, and such a covering is called a $p$-gonal morphism. The $p$-gonal Riemann surfaces have been extensively studied, see $[\mathbf{1}],[\mathbf{2}],[\mathbf{6}],[\mathbf{8}],[\mathbf{9}],[\mathbf{1 2}]$ and $[\mathbf{1 3}]$. A $p$-gonal Riemann surface is called real $p$ gonal if there is an anticonformal involution (symmetry) $\sigma$ of $X$ commuting with the $p$-gonal morphism.

Let $X_{g}$ be a real $p$-gonal Riemann surface of genus $g \geq 2$. A symmetry $\sigma$ of $X_{g}$ is an anticonformal involution of $X_{g}$. The topological type of a symmetry is determined by the number of connected components, called ovals, of the fixed-point set Fix $(\sigma)$ and the orientability of the Klein surface $X /\langle\sigma\rangle$. We say that $\sigma$ has species $\Sigma_{\sigma}=+k$ if $\operatorname{Fix}(\sigma)$ consists of $k$ ovals and $X /\langle\sigma\rangle$ is orientable, and $\Sigma_{\sigma}=-k$ if Fix $(\sigma)$ consists of $k$ ovals and $X /\langle\sigma\rangle$ is non-orientable. The set $\operatorname{Fix}(\sigma)$ corresponds to the real part of a complex algebraic curve representing $X$, which admits an equation with real coefficients.

If the $p$-gonal morphism is a cyclic regular covering, then the Riemann surface is called real cyclic $p$-gonal. When $p=2$ the surface $X_{g}$ is called hyperelliptic. A Riemann surface represented by an algebraic curve given 
by an equation of the form

$$
y^{p}=\prod\left(x-a_{i}\right) \prod\left(x-b_{j}\right)^{2} \cdots \prod\left(x-m_{j}\right)^{p-1}
$$

where the coefficients of the polynomial $\prod\left(x-a_{i}\right) \cdots \prod\left(x-m_{j}\right)^{p-1}$ are real is a real cyclic $p$-gonal Riemann surface. The complex conjugation induces a symmetry on the above curve. A natural problem is to study and classify all possible symmetries of such a Riemann surface up to conjugacy, as they will produce non-isomorphic real models of the complex algebraic curve.

In Section 2 we characterise real cyclic $p$-gonal Riemann surfaces, where $p$ is an odd prime, in terms of signatures of Fuchsian and NEC groups. In Section 3 we determine all possible symmetries of a real cyclic $p$-gonal Riemann surface represented by an algebraic curve with equation (1.1).

\section{Signatures of real cyclic $p$-gonal Riemann surfaces.}

Let $X_{g}$ be a compact Riemann surface of genus $g \geq 2$. The surface $X_{g}$ can be represented as a quotient $X_{g}=\mathcal{H} / \Gamma$ of the upper half plane $\mathcal{H}$ under the action of a surface Fuchsian group $\Gamma$, that is, a cocompact orientationpreserving subgroup of the group $\mathcal{G}=\operatorname{Aut}(\mathcal{H})$ of conformal and anticonformal automorphisms of $\mathcal{H}$ without elliptic elements. A discrete, cocompact subgroup $\Gamma$ of $\operatorname{Aut}(\mathcal{H})$ is called an NEC (non-euclidean crystallographic) group. The subgroup of $\Gamma$ consisting of the orientation-preserving elements is called the canonical Fuchsian subgroup of $\Gamma$, it is denoted by $\Gamma^{+}$. The algebraic structure of an NEC group and the geometric structure of its quotient orbifold are given by the signature of $\Gamma$ :

$$
s(\Gamma)=\left(h, \pm,\left[m_{1}, \ldots, m_{r}\right],\left\{\left(n_{11}, \ldots, n_{1 s_{1}}\right), \ldots,\left(n_{k 1}, \ldots, n_{k s_{k}}\right)\right\}\right) .
$$

The orbit space $\mathcal{H} / \Gamma$ is an orbifold with underlying surface of genus $h$, having $r$ cone points and $k$ boundary components, each with $s_{j} \geq 0$ corner points. The signs " + " and " - " correspond to orientable and non-orientable orbifolds respectively. The integers $m_{i}$ are called the proper periods of $\Gamma$ and they are the orders of the cone points of $\mathcal{H} / \Gamma$. The brackets $\left(n_{i 1}, \ldots, n_{i s_{i}}\right)$ are the period cycles of $\Gamma$ and the integers $n_{i j}$ are the link periods of $\Gamma$ and the orders of the corner points of $\mathcal{H} / \Gamma$. The group $\Gamma$ is called the fundamental group of the orbifold $\mathcal{H} / \Gamma$.

A group $\Gamma$ with signature (2.1) has a canonical presentation with generators:

$$
\begin{gathered}
x_{1}, \ldots, x_{r}, e_{1}, \ldots, e_{k}, c_{i j}, 1 \leq i \leq k, 1 \leq j \leq s_{i}+1, \text { and } \\
a_{1}, b_{1}, \ldots, a_{h}, b_{h}
\end{gathered}
$$

if $\mathcal{H} / \Gamma$ is orientable, or

$$
d_{1}, \ldots, d_{h}
$$


otherwise, and relators:

$$
\begin{gathered}
x_{i}^{m_{i}}, \quad i=1, \ldots, r \\
c_{i j}^{2},\left(c_{i j-1} c_{i j}\right)^{n_{i j}}, c_{i 0} e_{i}^{-1} c_{i s_{i}} e_{i}, \quad i=1, \ldots, k, j=2, \ldots, s_{i}+1
\end{gathered}
$$

and $x_{1} \cdots x_{r} e_{1} \cdots e_{k} a_{1} b_{1} a_{1}^{-1} b_{1}^{-1} \cdots a_{h} b_{h} a_{h}^{-1} b_{h}^{-1}$ or $x_{1} \cdots x_{r} e_{1} \cdots e_{k} d_{1}^{2} \cdots d_{h}^{2}$ according to whether $\mathcal{H} / \Gamma$ is orientable or not. This last relation is called the long relation.

The hyperbolic area of the orbifold $\mathcal{H} / \Gamma$ coincides with the hyperbolic area of an arbitrary fundamental region of $\Gamma$ and equals:

$$
\mu(\Gamma)=2 \pi\left(\varepsilon h-2+k+\sum_{i=1}^{r}\left(1-\frac{1}{m_{i}}\right)+\frac{1}{2} \sum_{i=1}^{k} \sum_{j=1}^{s_{i}}\left(1-\frac{1}{n_{i j}}\right)\right)
$$

where $\varepsilon=2$ if there is a ${ }^{\prime \prime}+{ }^{\prime \prime} \operatorname{sign}$ and $\varepsilon=1$ otherwise. If $\Gamma^{\prime}$ is a subgroup of $\Gamma$ of finite index then $\Gamma^{\prime}$ is an NEC group and the following Riemann-Hurwitz formula holds:

$$
\left[\Gamma: \Gamma^{\prime}\right]=\mu\left(\Gamma^{\prime}\right) / \mu(\Gamma) .
$$

An NEC group $\Gamma$ without elliptic elements is called a surface group and it has signature $(h ; \pm ;[-],\{(-), . k .,(-)\})$. In such a case $\mathcal{H} / \Gamma$ is a Klein surface, i.e., a surface with a dianalytic structure of topological genus $h$, orientable or not according to the sign " $+{ }^{\prime \prime}$ or " $-{ }^{\prime \prime}$, and having $k$ boundary components. Conversely, a Klein surface whose complex double has genus greater than one can be expressed as $\mathcal{H} / \Gamma$ for some NEC surface group $\Gamma$. Furthermore, given a Riemann (resp. Klein) surface represented as the orbit space $X=\mathcal{H} / \Gamma$, with $\Gamma$ a surface group, a finite group $G$ is a group of automorphisms of $X$ if and only if there exists an NEC group $\Delta$ and an epimorphism $\theta: \Delta \rightarrow G$ with $\operatorname{ker}(\theta)=\Gamma$ (see [5]). The NEC group $\Delta$ is the lifting of $G$ to the universal covering $\pi: \mathcal{H} \rightarrow \mathcal{H} / \Gamma$ and is called the universal covering transformation group of $(X, G)$.

Definition 1. For a prime $p$, a real cyclic $p$-gonal Riemann surface is a triple $(X, f, \sigma)$ where $\sigma$ is a symmetry of $X, f$ is a cyclic $p$-gonal morphism and $f \circ \sigma=c \circ f$, and $c$ is the complex conjugation.

Notice that by Lemma 2.1 in [1] the condition $f \circ \sigma=c \circ f$ is automatically satisfied for genera $g \geq(p-1) 2+1$, since the $p$-gonal morphism is unique. From now on, the genera will satisfy the condition above. As a consequence of the assumption $g \geq(p-1) 2+1$ for the genera of the $p$-gonal surface $X_{g}$ we have that the group $C_{p}$ generated by the $p$-gonal morphism is a normal subgroup of $\mathrm{Aut}^{+}\left(X_{g}\right)$. Notice the the classification method fails for surfaces with genera in the range $2 \leq g \leq(p-1) 2$. For instance, there are two 7-gonal surfaces of genus 3 . One of them, $X_{3}$, is the Klein's quartic with $\operatorname{Aut}^{+}\left(X_{3}\right) \simeq P S L_{2}(7)$, in this case $C_{7}$ is non-normal in $P S L_{2}(7)$. 
We give now a characterisation of real cyclic $p$-gonal Riemann surfaces represented by real equations via NEC groups.

Theorem 1 ([7]). Let $X$ be a Riemann surface with genus $g$. The surface $X$ admits a symmetry $\sigma$ and a meromorphic function $f$ such that $(X, f, \sigma)$ is a real cyclic p-gonal Riemann surface represented by a curve with real equation $y^{p}=\prod\left(x-a_{i}\right) \cdots \prod\left(x-m_{j}\right)^{p-1}$ if and only if there are an NEC group $\Delta$ with signature $(0,+,[p, \ldots, p],\{(p, \ldots, p)\})$ and an epimorphism $\theta: \Delta \rightarrow D_{p}$ such that $X$ is conformally equivalent to $\mathcal{H} / \operatorname{Ker} \theta$ and $\operatorname{Ker} \theta$ is an NEC Fuchsian surface group.

Let $(X, f, \sigma)$ be a real cyclic $p$-gonal Riemann surface uniformised by a Fuchsian surface group $\Gamma$. Consider the automorphism $\varphi: X \rightarrow X$ such that $X /\langle\varphi\rangle$ is the Riemann sphere and $\varphi$ is a deck-transformation of the covering $f$. Notice that the group $\Delta$ is the universal covering transformation group of $(X, \varphi, \sigma)$, that $D_{p}=\langle\varphi, \sigma\rangle$ and that the canonical Fuchsian subgroup $\Delta^{+}$ is the universal covering transformation group of $(X, \varphi)$. Thus $X /\langle\varphi\rangle$ is a sphere with conic points of order $p$. Let $\bar{\sigma}$ be the symmetry in the Riemann sphere $X /\langle\varphi\rangle$ induced by $\sigma$. Since the triple $(X, \varphi, \sigma)$ is represented by the equation $y^{p}=\prod\left(x-a_{i}\right) \cdots \prod\left(x-m_{j}\right)^{p-1}$, the symmetry $\sigma$ is given by the map $\sigma: X \rightarrow X$ defined by $\sigma:(x, y) \rightarrow(\bar{x}, \bar{y})$. The set of real solutions of 1.1 is the set Fix $(\sigma)$. Thus $\bar{\sigma}$ is conjugated to the complex conjugation. Then $X /\langle\varphi, \sigma\rangle=X /\langle\varphi\rangle /\langle\bar{\sigma}\rangle$ is a disc with corner(s) and conic points of order $p$.

With the above notation:

Theorem 2. Let $X$ be a real cyclic p-gonal Riemann surface such that $\langle\varphi, \sigma\rangle$ is isomorphic to $D_{p}$. If $G$ is the group of conformal and anticonformal automorphisms of $X$, then $X / G$ is uniformised by an NEC group $\Lambda$ such that there is a surface Fuchsian subgroup $\Gamma \leq \Lambda$ uniformising $X$ and the group $\Lambda$ has one of the following signatures:

$$
\begin{aligned}
& \left(0,+,[\overbrace{p, \ldots, p}^{r}, q p^{\epsilon}],\{(\overbrace{p, \ldots, p}^{s})\}\right), \text { where } \epsilon=0 \text { or } 1 \text { and } \\
& 2 r+s=\frac{2 g+2(1-\epsilon)(p-1)}{q(p-1)} . G /\langle\varphi\rangle=C_{q} \times C_{2} .
\end{aligned}
$$

$$
\begin{aligned}
& \left(0,+,[\overbrace{p, \ldots, p}^{r}],\left\{(q p^{\epsilon_{1}}, \overbrace{p, \ldots, p}^{s_{1}}, q p^{\epsilon_{2}}, \overbrace{p, \ldots, p}^{s_{2}})\right\}\right), \text { where } \epsilon_{i}=0 \text { or } 1 \text { and } \\
& 2 r+s_{1}+s_{2}=\frac{2 g+\left(2-\epsilon_{1}-\epsilon_{2}\right)(p-1)}{q(p-1)} . G /\langle\varphi\rangle=D_{q} .
\end{aligned}
$$

(III) $\left(0,+,[\overbrace{p, \ldots, p}^{r}, 2 p^{\epsilon_{1}}],\left\{(q p^{\epsilon_{2}}, \overbrace{p, \ldots, p}^{s})\right\}\right)$, where $\epsilon_{i}=0$ or 1 and $2 r+s=$ $\frac{g+\left(1-q \epsilon_{1}-\epsilon_{2}\right)(p-1)}{q(p-1)} . G /\langle\varphi\rangle=D_{q} \rtimes C_{2}$.

(IV) $\left(0,+,[\overbrace{p, \ldots, p}^{r}],\left\{(2 p^{\epsilon_{1}}, \overbrace{p, \ldots, p}^{s_{1}}, 2 p^{\epsilon_{2}}, \overbrace{p, \ldots, p}^{s_{2}}, q p^{\epsilon_{3}}, \overbrace{p, \ldots, p}^{s_{3}})\right\}\right)$, where $\epsilon_{i}=0$ or 1 and $2 r+s_{1}+s_{2}+s_{3}=\frac{2 g+\left(2-q \epsilon_{1}-q \epsilon_{2}-2 \epsilon_{3}\right)(p-1)}{2 q(p-1)}$. $G /\langle\varphi\rangle=D_{q} \times C_{2}$. 
(V) $\left(0,+,[\overbrace{p, \ldots, p}^{r}],\left\{(2 p^{\epsilon_{1}}, \overbrace{p, \ldots, p}^{s_{1}}, 3 p^{\epsilon_{2}}, \overbrace{p, \ldots, p}^{s_{2}}, 3 p^{\epsilon_{3}}, \overbrace{p, \ldots, p}^{s_{3}})\right\}\right)$, where $\epsilon_{i}=0$ or 1 and $2 r+s_{1}+s_{2}+s_{3}=\frac{g+\left(1-3 \epsilon_{1}-2 \epsilon_{2}-2 \epsilon_{3}\right)(p-1)}{6(p-1)}$. $G /\langle\varphi\rangle=S_{4}$.

(VI) $\left(0,+,[\overbrace{p, \ldots, p}^{r}, 3 p^{\epsilon_{1}}],\left\{(2 p^{\epsilon_{2}}, \overbrace{p, \ldots, p}^{s})\right\}\right)$, where $\epsilon_{i}=0$ or 1 and $2 r+s=$ $\frac{g+\left(1-4 \epsilon_{1}-3 \epsilon_{2}\right)(p-1)}{6(p-1)} . G /\langle\varphi\rangle=A_{4} \rtimes C_{2}$.

(VII) $\left(0,+,[\overbrace{p, \ldots, p}^{r}],\left\{(2 p^{\epsilon_{1}}, \overbrace{p, \ldots, p}^{s_{1}}, 3 p^{\epsilon_{2}}, \overbrace{p, \ldots, p}^{s_{2}}, 4 p^{\epsilon_{3}}, \overbrace{p, \ldots, p}^{s_{3}})\right\}\right)$, where $\epsilon_{i}=0$ or 1 and $2 r+s_{1}+s_{2}+s_{3}=\frac{g+\left(1-6 \epsilon_{1}-4 \epsilon_{2}-3 \epsilon_{3}\right)(p-1)}{12(p-1)}$. $G /\langle\varphi\rangle=S_{4} \times C_{2}$.

(VIII) $\left(0,+,[\overbrace{p, \ldots, p}^{r}],\left\{(2 p^{\epsilon_{1}}, \overbrace{p, \ldots, p}^{s_{1}}, 3 p^{\epsilon_{2}}, \overbrace{p, \ldots, p}^{s_{2}}, 5 p^{\epsilon_{3}}, \overbrace{p, \ldots, p}^{s_{3}})\right\}\right)$, where $\epsilon_{i}=0$ or 1 and $2 r+s_{1}+s_{2}+s_{3}=\frac{g+\left(1-15 \epsilon_{1}-10 \epsilon_{2}-6 \epsilon_{3}\right)(p-1)}{30(p-1)}$. $G /\langle\varphi\rangle=A_{5} \times C_{2}$.

Notice that in cases (VII) and (VIII) the factor group $C_{2}$ of $G /\langle\varphi\rangle$ is generated by the antipodal map.

Proof. Consider the chain of coverings $X=\mathcal{H} / \Gamma \rightarrow X /\langle\varphi\rangle=\mathcal{H} / \Delta^{+} \rightarrow$ $X / G=\mathcal{H} / \Lambda$ with uniformising groups $\Gamma \leq \Delta^{+} \leq \Lambda$, where $s\left(\Delta^{+}\right)=$ $(0,+,[\overbrace{p, \ldots, p}],\{\})$ and $s(\Lambda)=\left(h, \pm,\left[m_{1}, \ldots, m_{r}\right],\left\{\left(n_{11}, \ldots, n_{1 s_{1}}\right), \ldots\right.\right.$, $\left.\left.\left(n_{k 1}, \ldots, n_{k s_{k}}\right)\right\}\right)$. Furthermore by Lemma 2.1 in [1] the group $\langle\varphi\rangle$ is a normal subgroup of $G$. By Theorem 1 the factor group $\bar{G}=G /\langle\varphi\rangle$ is a finite group of conformal and anticonformal automorphisms of the Riemann sphere. (See also [12].)

In other words, we have an epimorphism $\theta: \Lambda \rightarrow \bar{G}$ with $\operatorname{Ker} \theta=\Delta^{+}$. This yields the signature of the group $\Lambda$ in terms of the signature of $\Delta^{+}$ and the group $\bar{G}$. Let $p_{i}$ and $q_{i j}$ be the orders in $\bar{G}$ of $\theta\left(x_{i}\right)$ and $\theta\left(c_{i j-1} c_{i j}\right)$ respectively, where $x_{i}, c_{i j}$ are generators in the canonical presentation of $\Lambda$ associated to the signature (2.1). By [3] and [5] each proper period $m_{i}$ induces $\frac{|\bar{G}|}{p_{i}}$ proper periods $\frac{m_{i}}{p_{i}}$ in $s\left(\Delta^{+}\right)$. Each link-period $n_{i j}$ induces $\frac{|\bar{G}|}{2 q_{i j}}$ proper periods $\frac{n_{i j}}{q_{i j}}$ in $s\left(\Delta^{+}\right)$. But $\frac{m_{i}}{p_{i}}=p$ or $\frac{m_{i}}{p_{i}}=1$ and $\frac{n_{i j}}{q_{i j}}=p$ or $\frac{n_{i j}}{q_{i j}}=1$, since $\Delta^{+}$is the group of the Riemann sphere with conic points of prime order $p$. We denote $K_{1}=\left\{i \quad \mid \frac{m_{i}}{p_{i}}=1\right\}, K_{p}=\left\{i \quad \mid \frac{m_{i}}{p_{i}}=p\right\}, H_{1}=\left\{(i, j) \quad \mid \frac{n_{i j}}{q_{i j}}=\right.$ $1\}$ and $H_{p}=\left\{(i, j) \quad \mid \frac{n_{i j}}{q_{i j}}=p\right\}$. Thus $\rho=\sum_{i \in K_{p}} \frac{|\bar{G}|}{p_{i}}+\sum_{(i, j) \in H_{p}} \frac{|\bar{G}|}{2 q_{i j}}$ 
Using the Riemann-Hurwitz formula $|\bar{G}|=\mu\left(\Delta^{+}\right) / \mu(\Lambda)$ we obtain

$$
\begin{aligned}
- & +\left(\sum_{i \in K_{p}} \frac{|\bar{G}|}{p_{i}}+\sum_{(i, j) \in H_{p}} \frac{|\bar{G}|}{2 q_{i j}}\right) \frac{(p-1)}{p} \\
= & |\bar{G}|(\alpha h-2+k)+\sum_{i \in K_{p}}|\bar{G}|\left(1-\frac{1}{p p_{i}}\right)+\sum_{i \in K_{1}}|\bar{G}|\left(1-\frac{1}{p_{i}}\right) \\
& +\sum_{(i, j) \in H_{p}} \frac{|\bar{G}|}{2}\left(1-\frac{1}{p q_{i j}}\right)+\sum_{(i, j) \in H_{1}} \frac{|\bar{G}|}{2}\left(1-\frac{1}{q_{i j}}\right),
\end{aligned}
$$

therefore $h=0, k=1, s(\Lambda)=\left(0,+,\left[p p_{1}, \ldots, p p_{r}\right],\left\{\left(p q_{1}, \ldots, p q_{s}\right)\right\}\right)$, where $p_{i}, q_{j} \in\{1, p\}$. By setting $K_{1}, K_{p}, H_{1}$ and $H_{p}$ in Equation (2.6) we obtain that $p_{i}, q_{j}$ satisfy the equation

$$
|\bar{G}|-2=\sum_{1}^{r}|\bar{G}|\left(1-\frac{1}{p_{i}}\right)+\sum_{1}^{s} \frac{|\bar{G}|}{2}\left(1-\frac{1}{q_{j}}\right) .
$$

To find $s(\Lambda)$ it is enough to find the nontrivial solutions of (2.7). We divide the study of (2.7) in eight cases according to the factor group $\bar{G}$ in the epimorphism $\theta: \Lambda \rightarrow \bar{G}$ with $\operatorname{Ker}(\theta)=\Delta^{+}$:

(I) $\bar{G}=C_{q} \times C_{2}$, where $C_{2}=\langle\bar{\sigma}\rangle$. The solution of Equation $(2.7)$ is $p_{1}=q$. Applying Riemann-Hurwitz formula to the covering $X \rightarrow X / G$ we obtain the signature $\left(0,+,[\overbrace{p, \ldots, p}^{r}, q p^{\epsilon}],\{(\overbrace{p, \ldots, p}^{s})\}\right)$, where $\epsilon=0$ or 1 and $2 r+s=$ $\frac{2 g+2(1-\epsilon)(p-1)}{q(p-1)}$.

(II) $\bar{G}=D_{q}$. The solution of $(2.7)$ is $q_{j_{1}}=q_{j_{2}}=q$. Therefore $s(\Lambda)=$ $\left(0,+,[\overbrace{p, \ldots, p}^{r}],\left\{(q p^{\epsilon_{1}}, \overbrace{p, \ldots, p}^{s_{1}}, q p^{\epsilon_{2}}, \overbrace{p, \ldots, p}^{s_{2}})\right\}\right)$, where $\epsilon_{i}=0$ or 1 and $2 r+$ $s_{1}+s_{2}=\frac{2 g+\left(2-\epsilon_{1}-\epsilon_{2}\right)(p-1)}{q(p-1)}$.

(III) $\bar{G}=D_{q} \rtimes C_{2}$. The solution of (2.7) is $p_{1}=2$, and $q_{1}=q$. Thus $s(\Lambda)$ becomes $\left(0,+,[\overbrace{p, \ldots, p}^{r}, 2 p^{\epsilon_{1}}],\left\{(q p^{\epsilon_{2}}, \overbrace{p, \ldots, p}^{s})\right\}\right)$, where $\epsilon_{i}=0$ or 1 and $2 r+s=\frac{g+\left(1-q \epsilon_{1}-\epsilon_{2}\right)(p-1)}{q(p-1)}$.

(IV) $\bar{G}=D_{q} \times C_{2}$. The solution in this case is $q_{j_{1}}=q_{j_{2}}=2$ and $q_{j_{3}}=q$. This yields $s(\Lambda)=\left(0,+,[\overbrace{p, \ldots, p}^{r}],\left\{(2 p^{\epsilon 1}, \overbrace{p, \ldots, p}^{s_{1}}, 2 p^{\epsilon_{2}}, \overbrace{p, \ldots, p}^{s_{2}}, q p^{\epsilon_{3}}, \overbrace{p, \ldots, p}^{s_{2}})\right\}\right)$, where $\epsilon_{i}=0$ or 1 and $2 r+s_{1}+s_{2}+s_{3}=\frac{2 g+\left(2-q \epsilon_{1}-q \epsilon_{2}-2 \epsilon_{3}\right)(p-1)}{2 q(p-1)}$. 
(V) $\bar{G}=S_{4}$. The solution of $(2.7)$ is $q_{j_{1}}=2, q_{j_{2}}=q_{j_{3}}=3$. Then $s(\Lambda)=$ $\left(0,+,[\overbrace{p, \ldots, p}^{r}],\left\{(2 p^{\epsilon_{1}}, \overbrace{p, \ldots, p}^{s_{1}}, 3 p^{\epsilon_{2}}, \overbrace{p, \ldots, p}^{s_{2}}, 3 p^{\epsilon_{3}}, \overbrace{p, \ldots, p}^{s_{3}})\right\}\right)$, where $\epsilon_{i}=0$ or 1 and $2 r+s_{1}+s_{2}+s_{3}=\frac{g+\left(1-3 \epsilon_{1}-2 \epsilon_{2}-2 \epsilon_{3}\right)(p-1)}{6(p-1)}$.

(VI) $\bar{G}=A_{4} \rtimes C_{2}$. The solution of (2.7) is $p_{1}=3$, and $q_{1}=2$. Thus $s(\Lambda)$ becomes $\left(0,+,[\overbrace{p, \ldots, p}^{r}, 3 p^{\epsilon_{1}}],\left\{(2 p^{\epsilon_{2}}, \overbrace{p, \ldots, p}^{s})\right\}\right)$, where $\epsilon_{i}=0$ or 1 and $2 r+s=\frac{g+\left(1-4 \epsilon_{1}-3 \epsilon_{2}\right)(p-1)}{6(p-1)}$.

(VII) $\bar{G}=S_{4} \times C_{2}$. The solution in this case is $q_{j_{1}}=2, q_{j_{2}}=3$ and $q_{j_{3}}=4$. This yields $s(\Lambda)=\left(0,+,[\overbrace{p, \ldots, p}^{r}],\left\{(2 p^{\epsilon_{1}}, \overbrace{p, \ldots, p}^{s_{1}}, 3 p^{\epsilon_{2}}, \overbrace{p, \ldots, p}^{s_{2}}, 4 p^{\epsilon_{3}}\right.\right.$, $\overbrace{p, \ldots, p}^{s_{3}})\})$, where $\epsilon_{i}=0$ or 1 and $2 r+s_{1}+s_{2}+s_{3}=\frac{g+\left(1-6 \epsilon_{1}-4 \epsilon_{2}-3 \epsilon_{3}\right)(p-1)}{12(p-1)}$. (VIII) $\bar{G}=A_{5} \times C_{2}$. The solution now is $q_{j_{1}}=2, q_{j_{2}}=3$ and $q_{j_{3}}=5$. This yields $s(\Lambda)=\left(0,+,[\overbrace{p, \ldots, p}^{r}],\left\{(2 p^{\epsilon_{1}}, \overbrace{p, \ldots, p}^{s_{1}}, 3 p^{\epsilon_{2}}, \overbrace{p, \ldots, p}^{s_{2}}, 5 p^{\epsilon_{3}}, \overbrace{p, \ldots, p}^{s_{2}})\right\}\right)$, where $\epsilon_{i}=0$ or 1 and $2 r+s_{1}+s_{2}+s_{3}=\frac{g+\left(1-15 \epsilon_{1}-10 \epsilon_{2}-6 \epsilon_{3}\right)(p-1)}{30(p-1)}$. This finishes the proof.

\section{Species of symmetries of real cyclic $p$-gonal Riemann surfaces.}

Let $X$ be a real cyclic $p$-gonal Riemann surface $X$ with real equation. In the next theorem we study the topological types of the possible real forms of $X$.

Theorem 3. Let $X$ be a real cyclic p-gonal Riemann surface with p-gonal automorphism $\varphi$ admitting a symmetry $\sigma$ with fixed points and such that $\langle\sigma, \varphi\rangle=D_{p}, p$ prime. If $\tau$ is another symmetry of $X$, then possible species of $\tau$ are (and all cases occur):

(1) $s(\Lambda)$ as in $(\mathrm{I})$.

a) $q \equiv 1 \bmod (2) . \Sigma_{\sigma}=\Sigma_{\tau}$. If $r+\epsilon>0$, then $\Sigma_{\sigma}=-1$. If $r+\epsilon=0$, then $\Sigma_{\sigma} \in\{-1,+1\}$.

b) $q \equiv 0 \bmod (2) . \Sigma_{\tau}=\Sigma_{\sigma}$ as in case (1a) or $\Sigma_{\tau}=0$.

(2) $s(\Lambda)$ as in (II).

a) $q \equiv 1 \bmod (2) . \Sigma_{\sigma}=\Sigma_{\tau}$ and $\Sigma_{\sigma}=-1$.

b) $q \equiv 0 \bmod (2), q \neq 2 . \Sigma_{\sigma}=-1$ and $\Sigma_{\tau}=-1$ or $\Sigma_{\tau}=+p,+1$.

(3) $s(\Lambda)$ as in (III). $\Sigma_{\tau}=0$ or $\Sigma_{\sigma}=\Sigma_{\tau}$, besides $\Sigma_{\sigma}=-1$.

(4) $s(\Lambda)$ as in (IV).

a) $q \equiv 1 \bmod (2) .\left\{\Sigma_{\sigma}, \Sigma_{\tau}\right\} \subset\left\{\Sigma_{1}, \Sigma_{2}\right\}$, where $\Sigma_{1} \in\{-1,+1,+p\}$. $\Sigma_{2} \in\{-1,+1,+p\}$. In both cases $\Sigma_{\sigma} \neq+p$ and $\Sigma_{\sigma} \neq+1$ if $\sigma$ is of the first type. 
b) $q \equiv 0 \bmod (2), q \neq 2 .\left\{\Sigma_{\sigma}, \Sigma_{\tau}\right\} \subset\left\{\Sigma_{1}, \Sigma_{2}, \Sigma_{3}, \Sigma_{4}\right\}$, where $\Sigma_{4}=0$, and $\Sigma_{2} \in\{-1,+1,+p\}$ and $\Sigma_{1}, \Sigma_{3} \in\{-1,+1,+p\}$. In all cases $\Sigma_{\sigma} \neq+p$ and $\Sigma_{\sigma}=+1$ if $\sigma$ is of the second type.

$q=2 . \quad\left\{\Sigma_{\sigma}, \Sigma_{\tau}\right\} \subset\left\{\Sigma_{1}, \Sigma_{2}, \Sigma_{3}, \Sigma_{4}\right\}$, where $\Sigma_{4}=0$, and $\Sigma_{i} \in$ $\{-1,+1,+p\}$ for $1 \leq i \leq 3$. In all cases $\Sigma_{\sigma} \neq+p$.

(5) $s(\Lambda)$ as in $(\mathrm{V}) . \Sigma_{\tau}=0$ or $\Sigma_{\sigma}=\Sigma_{\tau}$, with $\Sigma_{\sigma}=-1$.

(6) $s(\Lambda)$ as in (VI). $\Sigma_{\tau}=0$ or $\Sigma_{\sigma}=\Sigma_{\tau}$, with $\Sigma_{\sigma}=-1$.

(7) $s(\Lambda)$ as in (VII). $\left\{\Sigma_{\sigma}, \Sigma_{\tau}\right\} \subset\left\{\Sigma_{1}, \Sigma_{2}, 0\right\} . \Sigma_{1} \in\{-1,+1,+p\}$ and $\Sigma_{2} \in\{-1,+1,+p\}$, but $\Sigma_{\sigma}=-1$.

(8) $s(\Lambda)$ as in (VIII). $\Sigma_{\tau}=0$ or $\Sigma_{\sigma}=\Sigma_{\tau}$, with $\Sigma_{\sigma}=-1$.

Proof. Consider the following chain of epimorphisms: $\theta: \Lambda \stackrel{\bar{\theta}}{\rightarrow} G \stackrel{\phi}{\rightarrow} \bar{G}$. The symmetries of $X$ are symmetries $\tau$ in $G$ which are lifts of symmetries $\bar{\tau}$ in $\bar{G}$. The species of $\tau$ is given by the conjugacy classes of reflections in $\bar{\Lambda}=\bar{\theta}^{-1}\langle\varphi, \tau\rangle=\bar{\theta}^{-1} \phi^{-1}\langle\bar{\tau}\rangle=\theta^{-1}\langle\bar{\tau}\rangle$ and the orientability of $\mathcal{H} / \bar{\Lambda}$. Notice that $\langle\varphi, \tau\rangle$ is either a cyclic group $C_{2 p}$ or a dihedral group $D_{p}$ of order $2 p$.

As in Theorem 2 we divide the proof in eight cases corresponding to the different types of groups $\bar{G}$ of conformal and anticonformal automorphisms of the Riemann sphere. The signature of $\Lambda$ in each case is given by the corresponding case in Theorem 2.

(1a) $\bar{G}=C_{q} \times C_{2}, q \equiv 1 \bmod (2)$. In this case $\bar{G}$ contains just one conjugacy class of symmetries and so does $G$ : The one represented by $\sigma$. Moreover $D_{p}=\langle\varphi, \sigma\rangle$ is a normal subgroup of index $q$ in $G$. By [5] the signature of $\bar{\theta}^{-1}(\langle\varphi, \sigma\rangle)$ is $(0,+,[\overbrace{p, \ldots, p}^{r q+\epsilon}],\{(\overbrace{p, \ldots, p}^{q s})\})$. By [14] (see also [4] $) \Sigma_{\sigma}= \pm 1$ as $D_{p}=\langle\varphi, \sigma\rangle$. The sign + can only occur if $\bar{\theta}^{-1}(\langle\varphi, \sigma\rangle)$ has no proper periods, i.e., $r+\epsilon=0$. If $s=0$, then $r+\epsilon>0$, the possible species is -1 .

(1b) $\bar{G}=C_{q} \times C_{2}=\left\langle\hat{\rho}, \bar{\sigma} \mid \hat{\rho}^{q}, \bar{\sigma}^{2}, \hat{\rho}^{-1} \bar{\sigma} \hat{\rho} \bar{\sigma}\right\rangle$, with $q \equiv 0 \bmod (2)$. In this case $\bar{G}$ contains two conjugacy classes of symmetries, with representatives namely $\bar{\sigma}$ and $\hat{\rho}^{q / 2} \bar{\sigma}=\bar{\tau}$, and so does $G$. To find the species of the symmetries we have to consider the normal subgroups $\bar{\theta}^{-1}(\langle\varphi, \sigma\rangle)$ and $\bar{\theta}^{-1}(\langle\varphi, \tau\rangle)$ of $\Lambda$ with factor group $C_{q}$. By $[\mathbf{5}]$ they have signatures $(0,+,[\overbrace{p, \ldots, p}^{r q+\epsilon}],\{(\overbrace{p, \ldots, p}^{q s})\})$ and $(0,+[\overbrace{p, \ldots, p}^{r q+\epsilon+s q / 2}]$

and $(0,+,[\overbrace{p, \ldots, p}],\{-\})$ respectively. So species $\Sigma_{\sigma}$ is as in (1a) and $\Sigma_{\tau}=0$.

(2a) $\bar{G}=D_{q}=\left\langle\hat{\rho}, \bar{\sigma} \mid \hat{\rho}^{q}, \bar{\sigma}^{2},(\hat{\rho} \bar{\sigma}) 2\right\rangle$, with $q \equiv 1 \bmod (2)$. The group $\bar{G}$ contains one conjugacy class of symmetries and so does $G$. By the epimorphism $\theta: \Lambda \rightarrow D_{q}$ the images of reflections in $\Lambda$ leave one fixed coset in $D_{q}$, so 
we get that $\bar{\Lambda}_{\sigma}$ has signature $\left(0,+,[\overbrace{p, \ldots, p}^{r q+s_{1} \frac{q-1}{2}+s_{2}} \frac{q-1}{2}],\left\{(\overbrace{p, \ldots, p}^{s_{1}+s_{2}+\epsilon_{1}+\epsilon_{2}})\right\}\right)$. Now,
$s_{1}+s_{2}+r>0$ since $\Lambda$ is a NEC group, then $\Sigma_{\sigma}=-1$ by [4] and [14].

(2b) $\bar{G}=D_{q}=\left\langle\hat{\rho}, \bar{\sigma} \mid \hat{\rho}^{q}, \bar{\sigma}^{2},(\hat{\rho} \bar{\sigma})^{2}\right\rangle$, with $q \equiv 0 \bmod (2)$. The group $\bar{G}$ (and the group $G$ ) contains two conjugacy classes of symmetries, with representatives namely $\bar{\sigma}$ and $\hat{\rho} \bar{\sigma}=\bar{\tau}$. To find $\Sigma_{\sigma}$ and $\Sigma_{\tau}$ we have to study the images of reflections by an epimorphism $\theta: \Lambda \rightarrow D_{q}$. Each of these images leaves either $2 \bar{\sigma}$-cosets fixed and none from $\bar{\tau}$ or the other way round.

Thus the signatures of $\bar{\Lambda}_{\sigma}$ and $\bar{\Lambda}_{\tau}$ are $\left(0,+,[\overbrace{p, \ldots, p}^{r q+s_{1} \frac{q-2}{2}+s_{2} \frac{q}{2}}],\left\{(\overbrace{p, \ldots, p}^{\epsilon_{1}+2 s_{1}+\epsilon_{2}})\right\}\right)$ and rq $\overbrace{}^{\frac{q}{2}+s_{1}+s_{2} \frac{q-2}{2}} \overbrace{P, \ldots, p}^{\epsilon_{1}+2 s_{2}+\epsilon_{2}}$

$(0,+,[\overbrace{p, \ldots, p}],\{(\overbrace{p, \ldots, p})\})$. Now $\sigma$ has 1 oval and does not separate because $\bar{\theta}^{-1}(\langle\varphi, \sigma\rangle)$ contains proper periods since $s_{1}+s_{2}+r>0$ and $q>2$. If $\epsilon_{1}+s_{2}+\epsilon_{2}>0$, then $\langle\varphi, \tau\rangle=D_{p}$ and as before $\Sigma_{\tau}=-1$. If $\epsilon_{1}+s_{2}+\epsilon_{2}=0$ the signature of $\bar{\theta}^{-1}(\langle\varphi, \tau\rangle)$ becomes $\left(0,+,[\overbrace{p, \ldots, p}^{\left(2 r+s_{1}\right) \frac{q}{2}}],\{(-)\}\right)$. Thus $\Sigma_{\tau}=-1$, if $\langle\varphi, \tau\rangle=D_{p}$, and $\Sigma_{\tau}=+p,+1$ if $\langle\varphi, \tau\rangle=C_{2 p}$.

(3) $\bar{G}=D_{q} \rtimes C_{2}=\left\langle\bar{\rho}, \overline{\sigma_{1}}, \overline{\sigma_{2}} \mid \bar{\rho}^{2},{\overline{\sigma_{1}}}^{2},{\overline{\sigma_{2}}}^{2},\left(\overline{\sigma_{1} \sigma_{2}}\right)^{q}, \overline{\rho \sigma_{1} \rho \sigma_{2}}\right\rangle$. The group $\bar{G}$ (and $G$ ) contains two conjugacy classes of symmetries, with representatives namely $\bar{\sigma}=\bar{\sigma}_{1}$ and $\overline{\rho \sigma}=\bar{\tau}$. The images of reflections in $\Lambda$ are all mapped to conjugate reflections in $\bar{G}$. They are conjugate to $\bar{\sigma}$ as we know that $\sigma$ has fixed points. Thus $\Sigma_{\tau}=0$. On the other hand $\bar{\Lambda}_{\sigma}$ has always proper periods. Therefore $\Sigma_{\sigma}=-1$.

(4) $\bar{G}=D_{q} \times C_{2}=\left\langle\overline{\sigma_{1}}, \overline{\sigma_{2}}, \overline{\sigma_{3}} \mid{\overline{\sigma_{i}}}^{2},\left(\overline{\sigma_{1} \sigma_{2}}\right) 2,\left(\overline{\sigma_{2} \sigma_{3}}\right) 2,\left(\overline{\sigma_{3} \sigma_{1}}\right)^{q}\right\rangle$, with $\bar{\sigma}_{2}$ central in $\bar{G}$. First of all the group $\left\langle\varphi, \sigma_{2}\right\rangle$ is a normal subgroup of $G$ with factor group $D_{q}=\left\langle\bar{\sigma}_{1}, \bar{\sigma}_{3}\right\rangle$.

(4a) $q \equiv 1 \bmod (2)$. In this case $G$ has two conjugacy classes of reflections with representatives with images $\bar{\sigma}_{1}$ and $\bar{\sigma}_{2}$. Then there are two possible species for a symmetry of $X: \Sigma_{\sigma_{1}}, \Sigma_{\sigma_{2}}$. The possible signatures for $\bar{\theta}^{-1}\left(\left\langle\varphi, \sigma_{i}\right\rangle\right)$ are given by the epimorphism $\Lambda \stackrel{\hat{\theta}}{\rightarrow} D_{q}$. By this epimorphism the images of $c_{0}$ and $c_{s_{1}+i}$, for $i \geq 2$, are conjugated to $\overline{\sigma_{1}}$ and the image of $c_{1}, \ldots, c_{s_{1}+1}$ is the identity (representing the central symmetry). Therefore $c_{0}, c_{1}, c_{s+1}$ and $c_{s+2}$ fixes $q\left\langle\overline{\sigma_{2}}\right\rangle$-cosets and one $\left\langle\overline{\sigma_{1}}\right\rangle$-coset each, each $c_{1}, \ldots, c_{s_{1}+1}$ fixes $2 q\left\langle\overline{\sigma_{2}}\right\rangle$-cosets (and none $\left\langle\overline{\sigma_{1}}\right\rangle$-coset), and finally each $c_{s_{1}+i}, i \geq 2$ fixes two $\left\langle\overline{\sigma_{1}}\right\rangle$-cosets (and none $\left\langle\overline{\sigma_{1}}\right\rangle$-coset) in $\bar{G}$. Thus $\overline{\Lambda_{1}}$ and 
$\overline{\Lambda_{2}}$ have signatures

$$
\begin{aligned}
& \left(0,+,[\overbrace{p, \ldots, p}^{2 r q+q s_{1}+(q-1)\left(s_{2}+s_{3}\right)+\frac{q-1}{2}\left(\epsilon_{1}+\epsilon_{2}\right)}],\left\{(\overbrace{p, \ldots, p}^{\epsilon_{2}+2 s_{2}+2 \epsilon_{3}+\epsilon_{1}+2 s_{3}})\right\}\right) \text { and } \\
& \left(0,+,[\overbrace{p, \ldots, p}^{2 r q+q s_{2}+\epsilon_{3}+q s_{3}}],\left\{(\overbrace{p, \ldots, p}^{q \epsilon_{1}+2 q s_{1}+q \epsilon_{2}})\right\}\right), \text { see }[\mathbf{1 0}] .
\end{aligned}
$$

Altogether we have that $\Sigma_{1}$ is -1 if $s_{2}+s_{3}+\epsilon_{1}+\epsilon_{2}+\epsilon_{3}>0$, and $\Sigma_{1}$ is $+p,+1$ if $s_{2}+s_{3}+\epsilon_{1}+\epsilon_{2}+\epsilon_{3}=0$ and $\left\langle\varphi, \sigma_{1}\right\rangle$ is $C_{2 p}$. On the other hand $\Sigma_{2}$ is -1 if $s_{1}+\epsilon_{1}+\epsilon_{2}>0$ and and $r+s_{2}+s_{3}+\epsilon_{3}>0, \Sigma_{2}$ is +1 if $s_{1}+\epsilon_{1}+\epsilon_{2}>0$ and $r=s_{2}=s_{3}=\epsilon_{3}=0$, and finally $\Sigma_{2}$ is $+p,+1$ if $s_{1}+\epsilon_{1}+\epsilon_{2}=0$ and $\left\langle\varphi, \sigma_{2}\right\rangle=C_{2 p}$. In both cases $\Sigma_{\sigma} \neq+p$ since $\langle\varphi, \sigma\rangle=D_{p}$ and if $\sigma$ is conjugate to $\sigma_{1}$ then again $\Sigma_{\sigma} \neq+1$. No further restrictions exist.

(4b) $q \equiv 0 \bmod (2)$. In this case $G$ has four conjugacy classes of reflections with representatives with homomorphic images $\bar{\sigma}_{1}, \bar{\sigma}_{2}, \bar{\sigma}_{3}$ and $\left(\bar{\sigma}_{3} \bar{\sigma}\right)^{q / 2} \bar{\sigma}_{2}=$ $\bar{\sigma}_{4}$. Then there are four possible species for a symmetry of $X: \Sigma_{i}, 1 \leq$ $i \leq 4$. The species are also given by the epimorphism $\Lambda \stackrel{\hat{\theta}}{\rightarrow} D_{q}$. By this epimorphism the images of $c_{0}$ and $c_{s_{1}+s_{2}+i}$, for $i \geq 3$, are conjugated to $\overline{\sigma_{1}}$, the image of $c_{1}, \ldots, c_{s_{1}+1}$ is the identity (representing the central symmetry), and the images of $c_{s_{1}+i}$, for $2 \leq i \leq s_{2}+2$, are conjugate to $\overline{\sigma_{3}}$. First of all $\Sigma_{4}=0$ since no images of reflections by $\hat{\theta}$ are conjugate to $\bar{\sigma}_{4}$.

If $q=2$, then all the 3 symmetries are central and, as in (4a) the possible species for them are $-1,+1$ and $+p$.

If $q \neq 2$ then with the same procedure as in (4a) we get the following signatures for $\overline{\Lambda_{1}}, \overline{\Lambda_{2}}$ and $\overline{\Lambda_{3}}$ :

$$
\begin{aligned}
& \left.(0,+,[\overbrace{2 r q+q s_{2}+\epsilon_{3}+q s_{3}}^{2 r q+q\left(s_{1}+s_{2}\right)+\frac{q-2}{2} s_{3}+\frac{q-2}{2} \epsilon_{1}+\frac{q}{2} \epsilon_{2}}] \overbrace{\epsilon_{1}+2 q s_{1}+q \epsilon_{2}}],\left\{(\overbrace{p, \ldots, p}^{2 \epsilon_{3}+2 \epsilon_{1}+4 s_{3}})\right\}\right), \\
& \left.\left(0,+,[\overbrace{p, \ldots, p}^{2 r q+q\left(s_{1}+s_{3}\right)+\frac{q-2}{2} s_{2}+\frac{q-2}{2} \epsilon_{2}+\frac{q}{2} \epsilon_{1}})\right\}\right), \\
& \left(0,+,[\overbrace{p, \ldots, p}^{2 \epsilon_{3}+2 \epsilon_{2}+4 s_{2}}],\{(\overbrace{p, \ldots, p}^{p, \ldots, p})\}\right) .
\end{aligned}
$$

Both $\bar{\Lambda}_{1}$ and $\bar{\Lambda}_{3}$ must have proper periods because otherwise all parameters in the signature of $\Lambda$ except $\epsilon_{3}$ are 0 and then $\Lambda$ is a spherical group. Therefore $\Sigma_{1}$ is -1 if $s_{3}+\epsilon_{1}+\epsilon_{3}>0, \Sigma_{1}$ is $+p,+1$ if $s_{3}+\epsilon_{1}+\epsilon_{3}=0$ and $\left\langle\varphi, \sigma_{1}\right\rangle=C_{2 p} . \Sigma_{2}$ is -1 if $s_{1}+\epsilon_{1}+\epsilon_{2}>0$ and $r+s_{2}+s_{3}+\epsilon_{3}>0, \Sigma_{2}$ is +1 if $s_{1}+\epsilon_{1}+\epsilon_{2}>0$ and $r=s_{2}=s_{3}=\epsilon_{3}=0$, and finally $\Sigma_{2}$ is $+p,+1$ if $s_{1}+\epsilon_{1}+\epsilon_{2}=0$ and $\left\langle\varphi, \sigma_{2}\right\rangle=C_{2 p}$. Finally $\Sigma_{3}$ is -1 if $s_{2}+\epsilon_{2}+\epsilon_{3}>0$, $\Sigma_{3}$ is $+p,+1$ if $s_{2}+\epsilon_{2}+\epsilon_{3}=0$ and $\left\langle\varphi, \sigma_{3}\right\rangle=C_{2 p}$. In all cases $\Sigma_{\sigma} \neq+p$ since $\langle\varphi, \sigma\rangle=D_{p}$. Again $\Sigma_{\sigma} \neq+1$ if $\sigma$ is conjugate to $\sigma_{1}$ or $\sigma_{3}$. No further restrictions exist. 
(5) and (8) $\bar{G}=\left\langle\overline{\sigma_{1}}, \overline{\sigma_{2}}, \overline{\sigma_{3}} \mid{\overline{\sigma_{i}}}^{2},\left(\overline{\sigma_{1} \sigma_{2}}\right)^{2},\left(\overline{\sigma_{2} \sigma_{3}}\right)^{3},\left(\overline{\sigma_{3} \sigma_{1}}\right)^{q}\right\rangle$, where $q=3$ in (5) and $q=5$ in (8). $\bar{G}$, and thus $G$, contains two conjugacy classes of symmetries, with representatives namely $\bar{\sigma}=\bar{\sigma}_{1}$ and $\bar{\tau}$, with $\bar{\tau}$ conjugated to the antipodal map. Then $\Sigma_{\sigma}=\Sigma_{\sigma_{1}}$ and $\Sigma_{\tau}=0$. As in case (2a), by [10] and [14], given the epimorphism $\bar{\theta}$, all the generating reflections of $\Lambda$ induce reflections in $\bar{\theta}^{-1}\left(\left\langle\sigma_{1}\right\rangle\right)$. So $\Sigma_{\sigma}=-1$ as they induce also proper periods.

(6) $\bar{G}=A_{4} \rtimes C_{2}=\left\langle\bar{\rho}, \overline{\sigma_{1}}, \overline{\sigma_{2}} \mid \bar{\rho}^{3},{\overline{\sigma_{1}}}^{2},{\overline{\sigma_{2}}}^{2},\left(\overline{\sigma_{1} \sigma_{2}}\right)^{2}, \overline{\rho \sigma_{1} \rho \sigma_{2}}\right\rangle$, where $C_{2}$ is generated by the antipodal map. With the same arguments as in (3) we obtain that $G$ has two types of symmetries with representatives $\sigma$ and $\tau$ where $\Sigma_{\tau}=0$ and $\Sigma_{\sigma}=-1$.

(7) $\bar{G}=\left\langle\overline{\sigma_{1}}, \overline{\sigma_{2}}, \overline{\sigma_{3}} \mid{\overline{\sigma_{i}}}^{2},\left(\overline{\sigma_{1} \sigma_{2}}\right)^{2},\left(\overline{\sigma_{2} \sigma_{3}}\right)^{3},\left(\overline{\sigma_{3} \sigma_{1}}\right) 4\right\rangle=S_{4} \times C_{2}$. This case is as case $(4 \mathrm{~b})$ where the central symmetry is conjugated to the antipodal map and $\overline{\sigma_{1}}$ is conjugated to $\overline{\sigma_{2}}$. There are 3 conjugacy classes of symmetries with species $0, \Sigma_{1}=\Sigma_{\sigma_{3}}$ and $\Sigma_{2}=\Sigma_{\sigma_{2}}$. Now $\Sigma_{1}$ is -1 if $s_{3}+\epsilon_{1}+\epsilon_{3}>0$, $\Sigma_{1}$ is $+p,+1$ if $s_{3}+\epsilon_{1}+\epsilon_{3}=0$ and $\left\langle\varphi, \sigma_{3}\right\rangle$ is $C_{2 p}$. On the other hand $\Sigma_{2}$ is -1 if $s_{1}+s_{2}+\epsilon_{1}+\epsilon_{2}+\epsilon_{3}>0, \Sigma_{2}$ is $+p$ if $s_{1}+s_{2}+\epsilon_{1}+\epsilon_{2}+\epsilon_{3}=0$ and $\left\langle\varphi, \sigma_{2}\right\rangle=C_{2 p} . \Sigma_{\sigma} \neq+p,+1,0$ since $\langle\varphi, \sigma\rangle=D_{p}$ and $\sigma$ has fixed points.

To finish we show the existence of surfaces with the desired symmetries by listing appropriate groups $G$ and epimorphisms $\theta$. The $p$-gonal surfaces with the desired symmetries will be uniformised by the groups $\operatorname{Ker}(\theta)$. We distinguish the same eight cases as in Theorem 2.

(1) Let $G=\left\langle\varphi, \rho, \sigma \mid \varphi^{t}, \rho^{q}, \sigma^{2},(\varphi \sigma)^{2},(\varphi \rho)^{p q}, \rho^{-1} \sigma \rho \sigma\right\rangle$ and let $\theta: \Lambda \rightarrow G$ be defined by $\theta\left(x_{i}\right)=\varphi^{v_{i}}, 1 \leq i \leq r, \theta\left(x_{r+1}\right)=\rho \varphi^{\epsilon+v_{r+1}}, \theta\left(c_{2 j-1}\right)=\sigma$, $\theta\left(c_{2 j}\right)=\varphi \sigma, \theta(e)=\rho^{-1} \varphi^{l}$, where $j_{1}+\cdots+j_{r}+\epsilon+v_{r+1}+l \equiv 0 \bmod p$.

(2) Let $G=\left\langle\varphi, \tau, \sigma \mid \varphi^{p}, \sigma^{2}, \tau^{2},(\varphi \sigma)^{2},(\sigma \tau)^{q},(\varphi \tau)^{2}\right\rangle$. Let $\theta: \Lambda \rightarrow G$ be defined by $\theta\left(x_{i}\right)=\varphi^{v_{i}}, 1 \leq i \leq r, \theta\left(c_{0}\right)=\tau, \theta\left(c_{j}\right)=\sigma \varphi^{u_{j}}$ for $1 \leq j \leq$ $s_{1}+1$, where $u_{1}=\epsilon_{1}$ and $u_{j}=1-u_{j-1}, \theta\left(c_{j}\right)=\tau \varphi^{u_{j}}$, with $s_{1}+2 \leq j \leq$ $s_{1}+s_{2}+2$ where $u_{s_{1}+2}=\epsilon_{2}+1-u_{s_{1}+1}$ and $u_{j}=1-u_{j-1}, \theta(e)=\varphi^{l}$, where $v_{1}+\cdots+v_{r}+l \equiv 0 \bmod p$.

To obtain the species $\Sigma_{\tau}=+p,+1$ we consider groups $G$ with presentation $G=\left\langle\varphi, \tau, \sigma \mid \varphi^{p}, \sigma^{2}, \tau^{2},(\varphi \sigma)^{2},(\sigma \tau)^{q}, \varphi^{-1} \tau \varphi \tau\right\rangle$.

(3) Let $G=\left\langle\varphi, \rho, \tau, \sigma \mid \varphi^{p}, \rho^{2}, \tau^{2}, \sigma^{2}, \rho \sigma \rho \tau,(\varphi \rho)^{2}(\varphi \sigma)^{2},(\sigma \tau)^{q},(\varphi \tau)^{2}\right\rangle$. Let $\theta: \Lambda \rightarrow G$ be defined by $\theta\left(x_{i}\right)=\varphi^{v_{i}}, 1 \leq i \leq r, \theta\left(x_{r+1}\right)=\rho \varphi^{\epsilon_{1}+v_{r+1}}$, $\theta\left(c_{0}\right)=\tau, \theta\left(c_{j}\right)=\sigma \varphi^{u_{j}}$ for $1 \leq j \leq s_{1}+1$, where $u_{1}=\epsilon_{1}$ and $u_{j}=1-u_{j-1}$, $\theta(e)=\rho \varphi^{l}$, where $v_{1}+\cdots+v_{r+1}+\epsilon_{1}+l \equiv 0 \bmod p$.

(4) $G=\left\langle\varphi, \sigma_{1}, \sigma_{2}, \sigma_{3} \mid \sigma_{i}^{2},\left(\sigma_{1} \sigma_{2}\right)^{2},\left(\sigma_{2} \sigma_{3}\right)^{2},\left(\sigma_{3} \sigma_{1}\right)^{q}, \varphi^{p},\left(\varphi \sigma_{i}\right)^{2}\right\rangle$. Let $\theta: \Lambda \rightarrow$ $G$ be defined by $\theta\left(x_{i}\right)=\varphi^{v_{i}}, 1 \leq i \leq r, \theta\left(c_{0}\right)=\sigma_{1}, \theta\left(c_{j}\right)=\sigma_{2} \varphi^{u_{j}}$ for $1 \leq j \leq s_{1}+1$, where $u_{1}=\epsilon_{1}$ and $u_{j}=1-u_{j-1}, \theta\left(c_{j}\right)=\sigma_{3} \varphi^{u_{j}}$, with $s_{1}+2 \leq j \leq s_{1}+s_{2}+2$ where $u_{s_{1}+2}=\epsilon_{2}+1-u_{s_{1}+1}$ and $u_{j}=1-u_{j-1}$, $\theta\left(c_{j}\right)=\sigma_{1} \varphi^{u_{j}}$, with $s_{1}+s_{2}+3 \leq j \leq s_{1}+s_{2}+s_{3}+3$ where $u_{s_{1}+s_{2}+3}=$ 
$\epsilon_{3}+1-u_{s_{1}+s_{2}+2}$ and $u_{j}=1-u_{j-1}, \theta(e)=\varphi^{l}$, where $v_{1}+\cdots+v_{r}+l \equiv 0$ $\bmod p$.

To obtain the species $+p,+1$ one or two of the relations $\left(\varphi \sigma_{i}\right)^{2}$ in the presentation of $G$ must be substituted by relations $\varphi^{-1} \sigma_{i} \varphi \sigma_{i}$.

(5) and (8) $G=\left\langle\varphi, \sigma_{1}, \sigma_{2}, \sigma_{3} \mid \sigma_{i}^{2},\left(\sigma_{1} \sigma_{2}\right)^{2},\left(\sigma_{2} \sigma_{3}\right)^{3},\left(\sigma_{3} \sigma_{1}\right)^{q}, \varphi^{p},\left(\varphi \sigma_{i}\right)^{2}\right\rangle$, where $q=3$ in (5) and $q=5$ in (8) and let $\theta: \Lambda \rightarrow G$ be defined as in (4).

(6) $G=\left\langle\varphi, \rho, \sigma_{1}, \sigma_{2}\right| \varphi^{p}, \rho^{3}, \sigma_{1}^{2}, \sigma_{2}^{2},\left(\sigma_{1} \sigma_{2}\right)^{2}, \rho^{2} \sigma_{1} \rho \sigma_{2},\left(\varphi \sigma_{1}\right)^{2},\left(\varphi \sigma_{2}\right)^{2}$, $\left.(\varphi \rho)^{3 p}\right\rangle$. Let $\theta: \Lambda \rightarrow G$ be defined as $\theta\left(x_{i}\right)=\varphi^{v_{i}}, 1 \leq i \leq r, \theta\left(x_{r+1}\right)=$ $\rho \varphi^{\epsilon_{1}+v_{r+1}}, \theta\left(c_{0}\right)=\sigma_{1}, \theta\left(c_{j}\right)=\sigma_{2} \varphi^{u_{j}}$ for $1 \leq j \leq s+1$, where $u_{1}=\epsilon_{2}$ and $u_{j}=1-u_{j-1}, \theta(e)=\rho^{2} \varphi^{l}$, where $v_{1}+\cdots+v_{r+1}+\epsilon_{1}+l \equiv 0 \bmod p$.

(7) $G=\left\langle\varphi \sigma_{1}, \sigma_{2}, \sigma_{3} \mid \sigma_{i}^{2},\left(\sigma_{1} \sigma_{2}\right)^{2},\left(\sigma_{2} \sigma_{3}\right)^{3},\left(\sigma_{3} \sigma_{1}\right)^{4}, \varphi^{p},\left(\varphi \sigma_{i}\right)^{2}\right\rangle$ and let $\theta$ : $\Lambda \rightarrow G$ be defined as in (4). To obtain the species $+p,+1$ either the relations $\left(\varphi \sigma_{1}\right)^{2}$ and $\left(\varphi \sigma_{1}\right)^{2}$ or the relation $\left(\varphi \sigma_{3}\right)^{2}$ in the presentation of $G$ must be changed to the corresponding commuting relation.

The kernels of the above epimorphisms will uniformise surfaces with a symmetry with species -1 for general groups $\Lambda$. The same epimorphisms yield the species +1 in cases 1 and 4 under the restrictions on $\Lambda$ given in the first part of the theorem. Again, the same epimorphisms yield the species $+p,+1$ under the corresponding restrictions on $\Lambda$ given in the first part of the theorem.

The authors wish to thank to the referee for several helpful suggestions.

\section{References}

[1] R.D.M. Accola, On cyclic trigonal Riemann surfaces, I, Trans. Amer. Math. Soc., 283 (1984) 423-449, MR 85j:14052, Zbl 0584.14016.

[2] _ A classification of trigonal Riemann surfaces, Kodai Math. J., 23 (2000) 81-87, MR 2001b:14051, Zbl 0966.14021.

[3] E. Bujalance, Normal NEC signatures, Ill. J. Math., 26 (1982), 519-530, MR 84i:20049, Zbl 0483.20028.

[4] E. Bujalance, A.F. Costa and D. Singerman, Application of Hoare's theorem to symmetries of Riemann surfaces, Ann. Acad. Sci. Fenn., 18 (1993), 307-322, MR 94i:20087, Zbl 0794.30029.

[5] E. Bujalance, J.J. Etayo, J.M. Gamboa and G. Gromadzki, Automorphism Groups of Compact Bordered Klein Surfaces, Lect. Notes in Math., 1439, Springer-Verlag, Berlin, 1990, MR 92a:14018, Zbl 0709.14021.

[6] M. Cornalba, Systèmes pluricanoniques sur l'espace des modules des courbes et diviseurs de courbes $k$-gonales (d'après Harris et Mumford), Asterisque, 121-122 (1985), 7-24, MR 86c:14021, Zbl 0551.14014.

[7] A.F. Costa and M. Izquierdo, On real trigonal Riemann surfaces, submitted.

[8] G. González Díez, On prime Galois coverings of the Riemann sphere, Ann. Mat. Pura Appl., 168 (1995), 1-15, MR 97c:14033, Zbl 0846.30029. 
[9] B.H. Gross and J. Harris, Real algebraic curves, Ann. Scient. Ec. Norm. Sup., 14 (1981), 157-182, MR 83a:14028, Zbl 0533.14011.

[10] A.H.M. Hoare, Subgroups of NEC groups and finite permutation groups, Quart. J. Math. Oxford, 41 (1990), 45-59, MR 91d:20055, Zbl 0693.20044.

[11] A.H.M. Hoare and D. Singerman, The orientability of subgroups of plane groups, in 'Groups, St. Andrews', London Math. Soc. Lecture Note Ser., 71, Cambridge Univ. Press, Cambridge, 1982, 221-227, MR 85g:20061, Zbl 0489.20036.

[12] N. Ishii, Coverings over d-gonal curves, Tsukuba J. Math., 16 (1992), 173-189, MR 93g:14032, Zbl 0771.30042.

[13] _ Remarks on d-gonal cures, Tsukuba J. Math., 19 (1995), 329-345, MR 96k:14023, Zbl 0876.14019.

[14] M. Izquierdo and D. Singerman, Pairs of symmetries of Riemann surfaces, Ann. Acad. Sci. Fenn., 23 (1998), 3-24, MR 98m:20065, Zbl 0914.30029.

Received May 21, 2002 and revised May 8, 2003. The first author is partially supported by Ministerio de Ciencia y Tecnologia BFM2002-04801. The second author thanks the hospitality of UNED.

Depto. Matemáticas Fundamentales

UNED, C/ SENDA DEL REY 9

28040 MADRID

SPAIN

E-mail address: acosta@mat.uned.es

Matematiska Institutionen

LINKÖPINGS UNIVERSITET

58183 LINKÖPING

SWEDEN

E-mail address: miizq@mai.liu.se 